\title{
An Audit on Performance of Medical Students of the University of Kelaniya in Completing the Clinical Tasks during their Final Year Obstetrics \& Gynaecology Clinical Attachment
}

\author{
Palihawadana $\mathrm{TS}^{1}$, Motha $\mathrm{MBC}^{2}$, Dias $\mathrm{TD}^{2}$, Wijesinghe $\mathrm{PS}^{3}$
}

\section{INTRODUCTION}

Gaining skills in clinical tasks is an important aspect of learning activities of a medical student. Though students are exposed to clinical teaching from their third year of medical school, the final year provides an intense training period where emphasis is made on gaining such clinical skills. The students of the University of Kelaniya spend their final year as five clinical attachments at the university professorial units of the North Colombo Teaching hospital, rotating between the five specialities of medicine, surgery, paediatrics, obstetrics \& gynaecology and psychiatry. Except for a few formal teaching sessions on other disciplines, they spend most of the time learning and gaining competencies in the subjects they follow the clinical attachment.

During the clinical appointment of obstetrics \& gynaecology, the students are provided with learning objectives at the commencement. These include clinical tasks they are expected to complete during the appointment. While some are for procedures to be observed others are to be performed under supervision. At completion of each of these tasks the student is expected to obtain a signature from the clinical supervisor to certify completion. Such training techniques, with repetitive action, have shown to improve student skills development ${ }^{1}$.

\footnotetext{
1 Lecturer

Senior Lecturer

Senior Professor, Department of Obstetrics \& Gynaecology, University of Kelaniya.
}

Correspondence: Dr. T.S. Palihawadana

E-mail: thilipali@kln.ac.lk

Competing interests: None
The students undertake an objective structured clinical examination (OSCE) at the end of the clinical appointment. This is a component of the final assessment in obstetrics and gynaecology and is aimed at assessing the practical clinical skills of the students. This is comprised of 12 OSCE stations and assesses skills in counselling, communication, data interpretation and management of emergencies.

Learning objectives provide a positive reinforcement for learning and have proven to be an effective method of guidance in adult learning. The certification of competency is an accepted method of evaluating the level of achievement. Such methods are used in many undergraduate and postgraduate training programs.

As the completion of clinical tasks is not a mandatory requirement for progress in the training program, there is variation in the level of clinical tasks performed by the students. This audit was aimed at assessing the level of completion of these tasks by the students during their appointment. In addition to this the association of the level of completion and the performance at the OSCE by the students as well as their views on the reasons for not completion of the tasks were assessed.

\section{AUDIT STANDARDS}

1. Performing each clinical task at least once by a student- $100 \%$.

2. Achieving a total of at least $50 \%$ for all the tasks by a student $-100 \%$.

\section{OTHER OBJECTIVES}

1. To assess the association between level of completion of clinical tasks by the student and the performance at the OSCE
2. To describe the reasons for inability to complete the clinical tasks as perceived by the students.

\section{METHOD}

The audit was undertaken at the faculty of medicine, University of Kelaniya in October 2014. It was carried out on the day of the OSCE examination, which marks the end of their clinical appointment. A self-administered questionnaire was developed to collect the views of the students. This comprised of ten statements suggesting possible reasons for not being able to complete the tasks which included statements describing shortcomings in the system, the clinical supervisors as well as lack of motivation by the students (text box $1)$. The students were asked to mark their view on a 7-point Likert scale that ranged from strongly disagreeing to strongly agreeing which were scored from -3 to +3 .

Evaluation of the logbook is part of the end of clinical appointment assessment. The logbook has six parts and one of them is the list of clinical tasks they are expected to perform during their appointment. There are 39 clinical tasks (text box 2) and 5 repetitions of each task is desired. Therefore the maximum score that could be achieved was 195 and it was calculated to be a percentage. The OSCE assessment was marked by the academic staff members of the department at the conclusion of the examination. They were not aware of the score for the above clinical skills log or the students' views expressed.

The results are expressed as percentages. The association between the OSCE mark and the score for clinical skills log was determined by comparison of medians of the upper and lower tertiles using Mann-Whitney test. The views of 


\begin{tabular}{|ll|}
\hline \multicolumn{1}{|c|}{$\begin{array}{c}\text { Text Box 1. The statements given to students as possible explanations for } \\
\text { non-completion of clinical tasks list }\end{array}$} \\
\hline 1 & Learning objectives were not clear to me \\
2 & Instructions on the first day of the appointment were not clear to me \\
3 & The time was not adequate for me \\
4 & There was not enough clinical material in the ward \\
5 & The ward staff was not supportive \\
6 & The patients were not cooperative \\
7 & Clinical supervisors were not available to get signatures \\
8 & I did not think it is useful to do all that work \\
9 & I did not have enough self-motivation \\
10 & My learning methods are different to what was expected \\
\hline
\end{tabular}

the students on possible reasons for non-completion of tasks were scored according to the Likert scale score and the cumulative score for each statement was calculated. The head of the department approved the audit and permission to carry out the audit and the use of OSCE marks was granted by the dean of the faculty.

\section{RESULTS}

The audit included 37 students who sat the OSCE assessment on that day. All consented for participation and were included in the analysis.
None of the students had completed all tasks 5 times achieving a full score. No student had completed all tasks at least once. The best performance was 38 of 39 procedure performed at least once by one student while the worst was one student who had not done 28 of the 39 tasks at least once during the appointment. The number of students who obtained $50 \%$ or more for the clinical tasks was only $6(16 \%)$. None of the tasks were performed five times by all the students. There was no task that was not done by every student at least once. However, one task (task number 39) was not done at least once by 33 students and another (task number 16) by 31 students making them the least undertaken tasks. The average number of times performed for each task is shown in figure 1.

The mean OSCE mark for the students was $70.7 \%$ (SD 10.3). There was no correlation demonstrated between the score for completion of clinical tasks in the logbook and the performance at the OSCE assessment. The medians of the OSCE mark for upper and lower tertiles according to clinical task log score were 74.5 and $73.5(p=0.931)$, respectively.

The students' agreement or disagreement with each of the responses is shown in figure 2 . Of the possible reasons given to students, lack of time to complete the tasks (statement 3) was the factor most students agreed on. This was the only factor that had a positive cumulative score on the Likert scale. This was followed by the statements such as unavailability of the clinical supervisors (s7), thinking all that work is not needed (s8), lack of selfmotivation (s9) and the instructions at commencement being not clear (s2). The statements students mostly disagreed included patients not being cooperative (s6), thinking all that work is not needed (s8), lack of clinical material (s4) and ward staff not being supportive (s5).

\begin{tabular}{|ll|ll|ll|}
\hline \multicolumn{4}{|c|}{ Text Box 2. The clinical tasks to be performed / observed by the students during their clinical appointment } \\
\hline \multicolumn{2}{|c|}{ To be performed under supervision } & \multicolumn{2}{|c|}{ To be performed under supervision } & \multicolumn{2}{c|}{ To be observed } \\
\hline 1 & Take an obstetric history & 14 & Advice on contraception & 27 & A dating scan \\
2 & Take a gynaecological history & 15 & Write LSCS operation notes & A mid-trimester scan \\
3 & Obstetric examination & 16 & Removal of sutures & 29 & A fetal wellbeing scan \\
4 & Gynaecological examination & 17 & Venepuncture & 30 & Cervical smear test \\
5 & Assessment of Bishop score & 18 & Set up an intravenous drip & 31 & Taking a high vaginal swab \\
6 & Setting up an oxytocin infusion & 19 & Set up a blood transfusion & 32 & Insertion of a ring pessar \\
7 & Maintain a partogram & 20 & Do ward tests for urine albumin and sugar & 33 & Low forceps delivery \\
8 & Management of labour & 21 & Fill investigation forms & 34 & Vacuum delivery \\
9 & Repair of episiotomy & 22 & Prepare for gynaecology surgeries & 35 & Twin delivery \\
10 & Catheterization & 23 & Assist at gynae surgery & 36 & Breech delivery \\
11 & Prepare patients for LSCS & 24 & Write operation notes for minor surgeries & 37 & Manual removal of placenta \\
12 & Assist at LSCS & 25 & Write operation notes for major surgeries & 38 & Repair of vaginal / cervical tears \\
13 & Advice on postnatal exercise & 26 & Write diagnosis cards & 39 & Resuscitation of a shocked patient \\
& & &
\end{tabular}


Figure 1. The average number of times for each procedure done by the medical students during the clinical appointment

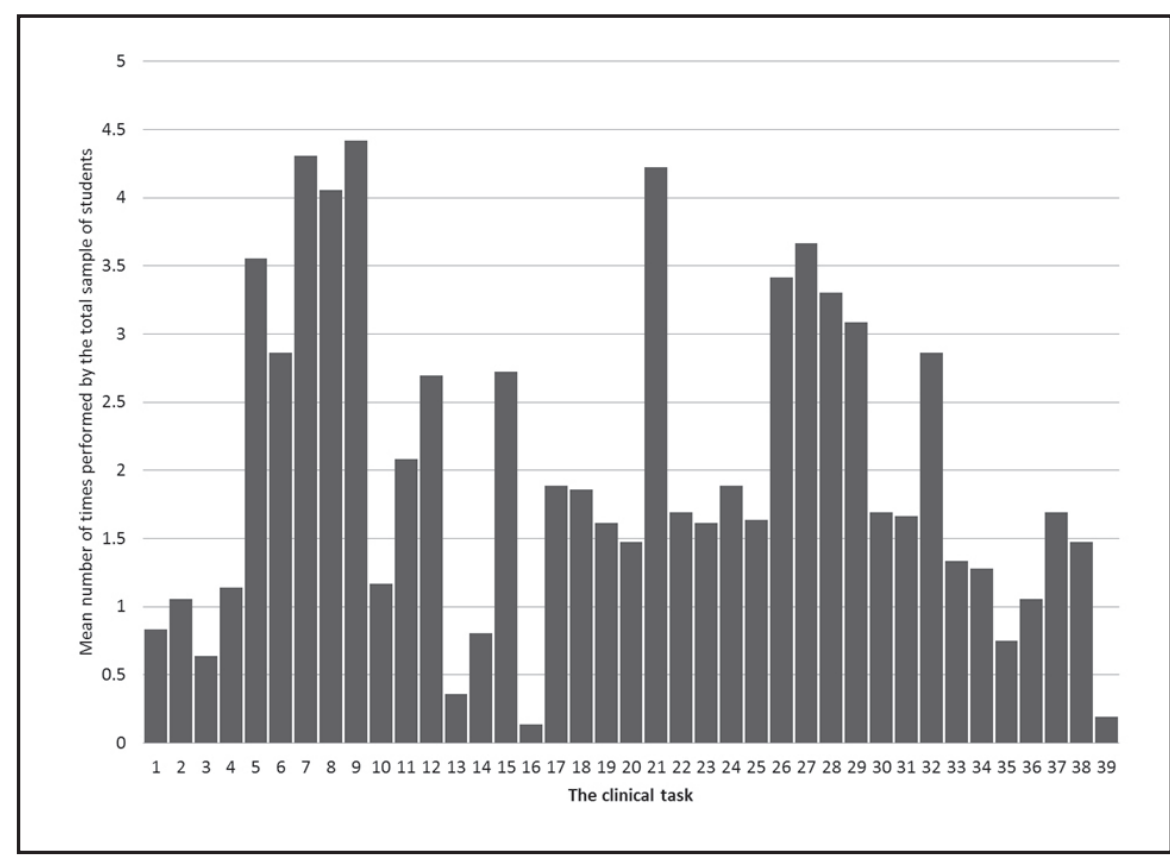

Figure 2. The cumulative score on the Likert scale for each statement as expressed by the total student sample

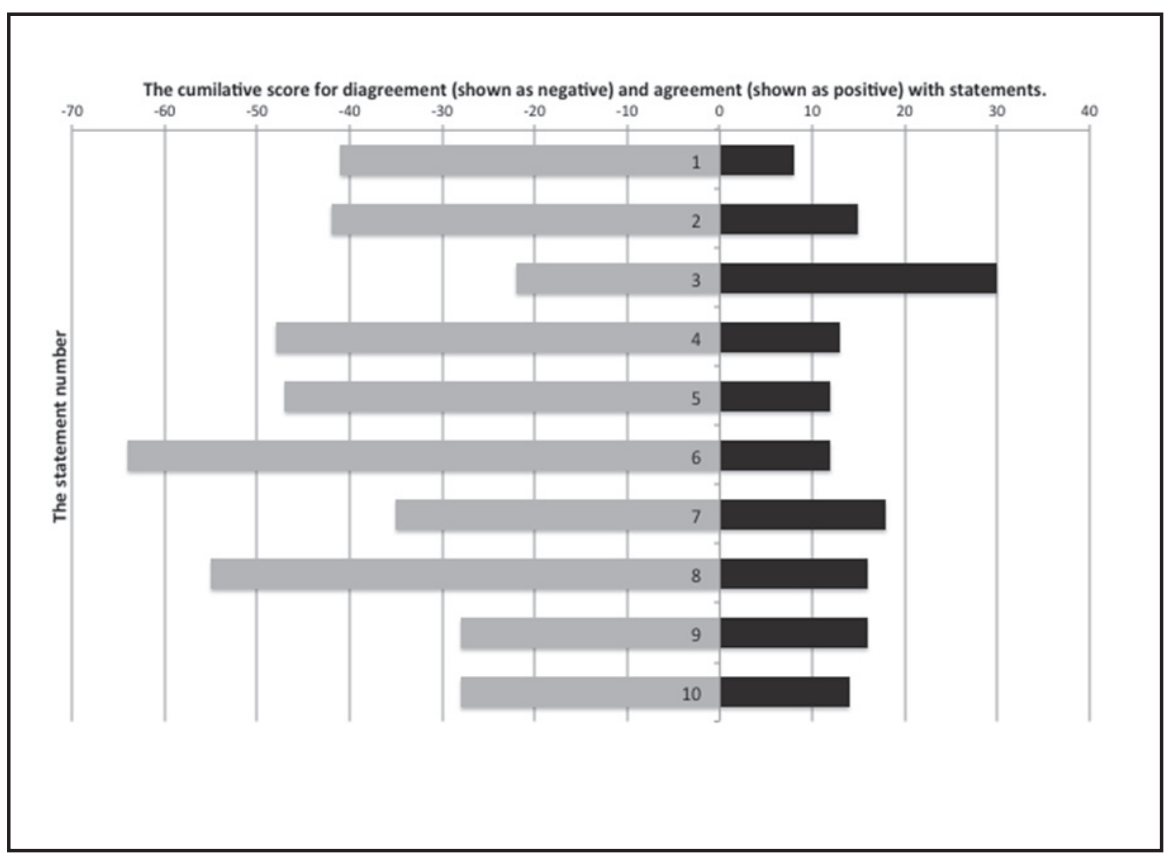

\section{DISCUSSION}

This audit was able to demonstrate some important aspects in the clinical appointment of medical students during their final year. Their completion of the clinical skills listed in the logbook was very unsatisfactory. They were far below the desired targets. None of the number of competency certification may have put a heavy burden on the clinical supervisors that is not easy to be met. Some tasks which the students undertake independently, such as filling up of investigation forms, may have made it difficult for them to obtain signatures since clinical supervisors will not be present at all times. Other effects can also co-exist, such as gender-based differences in training opportunities, which we did not assess in our study ${ }^{2}$.

We could not demonstrate any significant correlation between the level of completion in the clinical tasks and the marks obtained at the OSCE assessment. Previous studies have shown positive correlation with performance at assessment after a training program ${ }^{3}$. It is difficult to hypothesize the exact reasons for lack of such a relationship. The possible reasons could be that the log of work does not reflect the actual work undertaken by the student, the overall score of the clinical log does not correlate well with the clinical skills of the students while the possibility of the OSCE not assessing the clinical skills of a student also exist. Timing of the training, such as introduction of training early in the curriculum, also have an impact on development of other skills such as student confidence and team building skills ${ }^{4}$.

To our knowledge this is the first such audit performed among medical students to assess their level of completing clinical tasks and their attitude towards non-completion in a Sri Lankan setting. The findings of the audit were discussed at an audit meeting of the clinical department and further action was suggested to improve performance. These recommendations included review of the clinical tasks list and making the important skills mandatory. Such an approach would be compliant with the competency based training for essential skills. Also the number of repetitions required for each task should be critically looked at based on the requirement and the availability of clinical material. The place of other methods such as focused proficiency based training methods to teach skills, which have proven to be effective, 
also need to be explored ${ }^{5}$. We also hope to undertake an evaluation of our past OSCE questions with the help of a medical educationist to assess the degree of clinical skills assessment in previous examinations. We feel this audit provide valuable information for improvement of clinical appointment that can be used by different universities as well as in different specialities.

\section{REFERENCES}

1 Johnsen TS, Jacobsen G, Knoff T. The effect of practical training in obstetrics among medical students: symphysisfundal height measurements. Med Educ.1988;22:438-44.

2 Craig LB, Smith C, Crow SM, et al. Obstetrics and gynecology clerkship for males and females: similar curriculum, different outcomes? Med Educ Online. 2013;18:644. doi:10.1097/00001888199708000-00022

3 Homaifar N, Mwesigye D, Tchwenko S, et al. Emergency obstetrics knowledge and practical skills retention among medical students in Rwanda following a short training course. Int J Gynaecol Obstet. 2014;120:195-9. doi:10.1016/j. ijgo.2012.07.031

4 Kwiatkowski T, Rennie W, Fornari A, et al. Medical students as EMTs: skill building, confidence and professional formation. Med Educ Online. 2014;19:513. doi:10.3 109/0142159X.2012.638007

5 Gershuni V, Woodhouse J, Brunt LM. Retention of suturing and knot-tying skills in senior medical students after proficiency-based training: Results of a prospective, randomized trial. Surgery. 2013;154:823-30. doi:10.1016/j.surg. 2013.07.016 